\title{
Combinatorics on Finite Fields: the sign repartition for the quadratic residues
}

\author{
Şerban Bărcănescu
}

\begin{abstract}
In the present paper we present the equivalence between the combinatorial determination of the sign repartition for the quadratic residues and non-residues to the computation of the class number of certain quadratic extensions of the field of rationals.
\end{abstract}

\section{Introduction}

Let $p$ be a prime odd number and $g$ be a primitive root $\bmod p$, i.e. $g$ is a fixed generator of the multiplicative cyclic group of the prime field of characteristics $p$, denoted by $F_{p}^{*}$. In what follows we fix the canonical halbsystems of the "positive" $\operatorname{Pos}(\mathrm{p})=\left\{1,2, \ldots, \frac{p-1}{2}\right\}$ elements and of the "negative" $\operatorname{Neg}(\mathrm{p})=\left\{\frac{p+1}{2}, \ldots, p-1\right\}=\left\{-\frac{p-1}{2}, \ldots,-2,-1\right\}$ elements of the field $F_{p}$. We have the partition :

$F_{p}=\operatorname{Pos}(p) \cup\{0\} \cup N e g(p)$

Also, let $R=<g^{2 k} \mid k=0,1, \ldots, \frac{p-3}{2}>$ (residues) be the subgroup of the quadratic residues mod $p$ and $N=<g^{2 k+1} \mid k=0,1, \ldots, \frac{p-3}{2}>$ (non-residues) be its only residue class in $F_{p}^{*}$. We are interested in the repartition of the elements of Rand $N$ between $\operatorname{Pos}(\mathrm{p})$ and $\operatorname{Neg}(\mathrm{p})$ (a sample of such a situation is given by the well-known elementary proof of the gaussian criterion for the Legendre symbol). As such, the problem can not be solved with elementary

\footnotetext{
Key Words: quadratic residues, quadratic fields, class numbers

2010 Mathematics Subject Classification: Primary:11F20, 11R11, 11R29; Secondary:11B30

Received: October, 2013.

Revised: November, 2013.

Accepted: December, 2013.
} 
( and even not elementary) tools, but at least we can hope to solve the enumeration problem which naturally arises in this context.

\section{Results}

We begin with

Lemma 2.1. Let $d$ be a divisor of $p-1$ and $F_{p}^{*}=C_{0} \cup C_{1} \cup \ldots \cup \cup C_{d-1}$ be the partition of $F_{p}^{*}$ in residue classes after its unique subgroup $C_{0}$ of index $d$ .Then:

(i) for odd $d:-1 \in C_{0}$

(ii) for even $d:-1 \in C_{0}$ or $-1 \in C_{\frac{d}{2}}$

Proof. Let $-1 \in C_{h}$ for a certain $h(\bmod d)$. Then $-C_{h}=(-1) C_{h} \subset C_{h} . C_{h}=$ $C_{2 h}$ and since they have equal cardinality we have $-C_{h}=C_{2 h}$. By the same reason $-C_{2 h}=C_{3 h}$. But $-C_{2 h}=C_{h}$ because of the 2-periodicity of changing the sign (sign rule in a field). Then $C_{3 h}=C_{h}$ so $3 h \equiv h(\bmod d)$ and it follows $2 h \equiv 0(\bmod d)$. $\square$

In particular, for $d=2$ we have the :

Corollary 2.1. (erster erganzunssatz)

With the above notations:

$-1 \in R$ if $p \equiv 1(\bmod 4)$, therefore $-R=R$ and $-N=N$

$-1 \in N$ if $p \equiv 3(\bmod 4)$, therefore $-R=N$ and $-N=R \square$.

Let now $R^{+}=R \cap \operatorname{Pos}(p), R^{-}=R \cap N e g(p)$,so we have the partition :

(1) $R=R^{+} \cup R^{-}$

and similarly $N^{+}=N \cap \operatorname{Pos}(p), N^{-}=N \cap N e g(p)$, so we have the partition :

(2) $N=N^{+} \cup N^{-}$.

For simplicity we denote :

$a=\operatorname{card}\left(R^{+}\right)$and $b=\operatorname{card}\left(N^{+}\right)$.

Proposition 2.1. For $p \equiv 1(\bmod 4): a=b=\frac{p-1}{4}$.

Proof.

Multiplication by $(-1)$ on $F_{p}^{*}$ has a double efect in this case :

(i) -residue $=$ residue and - non residue $=$ non residue (Cor. 1 )

(ii) $-\operatorname{Pos}(p)=\operatorname{Neg}(p)$

Moreover, the multiplication by $(-1)$ is a permutation of $F_{p}^{*}$ so using (1) and (2), together with $a+b=\frac{p-1}{2}$ we get : 
$\operatorname{card}\left(R^{+}\right)=\operatorname{card}\left(R^{-}\right)=\operatorname{card}\left(N^{+}\right)=\operatorname{card}\left(N^{-}\right) \square$

So , in case $p \equiv 1(\bmod 4)$ we have a simple and complete answer concerning the enumeration of the sign repartition between the quadratic residues and non residues $\bmod p$.

However, in case $p \equiv 3(\bmod 4)$ we only have the relation :

$a+b=\frac{p-1}{2}$.

In order to determine $a$ and $b$ in this case we need another relation, the simplest of which would be an estimation of the difference $a-b$. Let us for the moment denote by $\Delta(p)$ this difference.

Making the computations in a mathematical package (for instance, under SAGE) we easily obtain the following estimations:

$\Delta(7)=1, \Delta(11)=\Delta(19)=\Delta(23)=\Delta(31)=\Delta(43)=\Delta(67)=\Delta(163)=$ $\Delta(307)=3, \Delta(47)=\Delta(79)=\Delta(103)=\Delta(127)=\Delta(179)=5, \Delta(71)=$ $\Delta(151)=\Delta(223)=7, \Delta(59)=\Delta(83)=\Delta(107)=\Delta(139)=\Delta(199)=$ $\Delta(211)=\Delta(283)=\ldots=\Delta(1423)=9, \Delta(131)=\Delta(227)=\Delta(239)=\ldots=$ $\Delta(111723)=15, \Delta(191)=\Delta(263)=13, \ldots, \Delta(599)=25, \ldots$, $\Delta(1019)=\Delta(1439)=39, \ldots, \Delta(1399)=27, \ldots, \Delta(1427)=45, \ldots, \Delta(1447)=$ $23 \ldots$.

As we can see, these results make plausible the following property:

(P) If $p \equiv 3(\bmod 4)$ then $\Delta(p)$ is positive and odd.

In fact, the property $(\mathrm{P})$ is true and more, we have the following remarkable result ([1] , ch.V, par.4,pag.346), formulated using the above notations and conventions:

Theorem 2.1. Let $p$ be a prime number such that $p \equiv 3(\bmod 4)$. Let $h=$ $\operatorname{ord} C l\left(\mathbb{Q}\left(\sqrt{p^{*}}\right)\right)$ be the class number of the quadratic number field generated by $p^{*}=(-1)^{\frac{p-1}{2}} p$.

Then :

(i) $h=a-b$ for $p \equiv 7(\bmod 8)$

(ii) $h=\frac{1}{3}(a-b)$ for $p \equiv 3(\bmod 8)$.

Moreover, $h$ is allways odd.

Solving the resulting linear system for $a$ and $b$, whose first equation is always :

$a+b=\frac{p-1}{2}$ and the second is given by the above Theorem, we obtain:

Proposition 2.2. The sign repartition enumeration for the quadratic residues and non residues modulo $p$ is :

(1) $a=b=\frac{p-1}{4}$ if $p \equiv 1(\bmod 8)$ or $p \equiv 5(\bmod 8)$

(ii.1) $a=\frac{p-1}{4}+\frac{3}{2} h, b=\frac{p-1}{4}-\frac{3}{2} h$ if $p \equiv 3(\bmod 8)$

(ii.2) $a=\frac{p-1}{4}+\frac{1}{2} h, b=\frac{p-1}{4}-\frac{1}{2} h$ if $p \equiv 7(\bmod 8)$

where his the class number of the quadratic number field $\mathbb{Q}(\sqrt{-p})$. 
Remark 2.1. Because of the linearity of the expresions above, we see that in fact the determination of the sign repartition enumeration is equivalent to the computation of the class number of the quadratic number field generated by $\sqrt{-p}$.

If we compute $h$ by using the values of $a$ and $b$ obtained from the system $a+b=\frac{p-1}{2}$ and the numerical values of $\Delta(p)=a-b$ listed above, the values of the class number $h$ result immediately and are in accordance to the usual tables of its values, e.g.:

$h(7)=1, h(11)=1, h(19)=1, h(23)=3, h(31)=341)=1, h(47)=$ $5, h(59)=3, h(167)=11, \ldots$,

$h(191)=13, h(599)=25, h(1019)=13, h(1439)=39$.

Acknowledgement.

The publication of this paper is supported by the grants PN-II-ID-WE2012-4-161 and PN-II-ID-WE-2012-4-169.

\section{References}

[1] Z.I.Borevitch and I.R.Schafarevich-Number Theory, Academic Press, (1966).

Şerban BĂRCĂNESCU

Simion Stoilow Institute of Mathematics of the Romanian Academy,

Research Unit 5, P.O. Box 1-764, Bucharest 014700, Romania

Email:Serban.Barcanescu@imar.ro 